\title{
A Multigrid Method for Nonconforming FE-Discretisations with Application to Non-Matching Grids
}

\author{
D. Braess, Bochum, M. Dryja, Warsaw, and W. Hackbusch, Leipzig
}

Received October 15, 1998

\begin{abstract}
Nonconforming finite element discretisations require special care in the construction of the prolongation and restriction in the multigrid process. In this paper, a general scheme is proposed, which guarantees the approximation property. As an example, the technique is applied to the discretisation by non-matching grids (mortar elements).

AMS Subject Classifications: 65F10, 65N55, 65N30.
\end{abstract}

Key Words: Nonconforming finite element method, mortar, multigrid method.

\section{Introduction}

Recently, domain decomposition methods have been applied to situations where subdomain meshes may be separately constructed and are non-matching along the interfaces. The method was called mortar element method in [3]. When this scheme is employed with finite elements, it may be considered as a nonconforming method or as a mixed method.

In this paper, we will treat the mortar elements in the framework of nonconforming methods, and we assume that the Lagrange multipliers have been eliminated as in the setting of the second author [9]. When multigrid methods are designed, there is now the problem that the finite element spaces are not nested.

Therefore, we have to construct appropriate prolongation operators. In the multigrid scheme for other elements, as, e.g., for the Crouzeix-Raviart elements in [5, $8]$ the $L_{2}$-projectors could be chosen for the prolongations. We will abandon this restriction and describe a more general framework which admits a lot of freedom in the construction. In particular, a prolongation that is natural for the mortar elements fits into our framework. The approximation property for the convergence proof will be derived from an auxiliary problem. In essence, we will only assume that an $L_{2}$ error estimate is known for the finite elements under consideration. 
In Section 2 we recall some notation for nonconforming finite elements. Section 3 is concerned with an extension of the prolongation operators which admits in Section 4 to derive the central approximation property from an $L_{2}$ error estimate. In Section 5 the associated smoothing property and the convergence is discussed. Section 6 provides the application to mortar elements in the geometric conforming case. We conclude with a generalisation to geometric nonconforming meshes.

After completing the paper, we learnt about the paper [11] investigating the mortar finite element method by other theoretical means.

\section{Multigrid Transfer}

\subsection{Variational Problem}

We consider a variational problem of the following form. Let $\mathcal{H}^{1}$ be a Hilbert space. Given a bilinear form $a(\cdot, \cdot)$ on $\mathcal{H}^{1} \times \mathcal{H}^{1}$ and a functional $f \in \mathcal{H}^{-1}:=\left(\mathcal{H}^{1}\right)^{\prime}$, we look for a solution $u \in \mathcal{H}^{1}$ of

$$
a(u, w)=f(w) \quad \text { for all } w \in \mathcal{H}^{1} .
$$

Let

$$
\mathcal{H}^{-1} \subset \mathcal{H}^{0} \subset \mathcal{H}^{1}
$$

be the Gelfand triple, e.g., $\mathcal{H}^{1}:=H_{0}^{1}(\Omega), \mathcal{H}^{0}:=L_{2}(\Omega)$, and $\mathcal{H}^{-1}:=H^{-1}(\Omega)$. In addition, we need a space $\mathcal{H}^{2} \subset \mathcal{H}^{1}$ (e.g., $\mathcal{H}^{2}=H^{2}(\Omega) \cap H_{0}^{1}(\Omega)$ ). The norms of $\mathcal{H}^{k}$ are denoted by $\|\cdot\|_{k}$. The scalar product in $\mathcal{H}^{0}$ is written as $(\cdot, \cdot)_{0}$.

We assume:

(i) Solvability: For all $f \in \mathcal{H}^{-1}$, (2.1) has a unique solution $u \in \mathcal{H}^{1}$ with $\|u\|_{1} \leq$ $C\|f\|_{-1}$.

(ii) Regularity: If $f \in \mathcal{H}^{0},(2.1)$ has a solution $u \in \mathcal{H}^{2}$ with $\|u\|_{2} \leq C\|f\|_{0}$.

\subsection{Nonconforming Discretisation}

Let $V_{\ell} \subset \mathcal{H}^{0}$ for $\ell=0,1, \ldots$ be a sequence of (nonconforming) finite element spaces, i.e., we do not assume that the spaces are nested. Instead of the bilinear form $a(\cdot, \cdot)$ a mesh-dependent bilinear form $a_{\ell}(\cdot, \cdot)$ on $V_{\ell} \times V_{\ell}$ is used. For $f \in \mathcal{H}^{0}$, (2.1) is discretised by

$$
u_{\ell} \in V_{\ell} \quad \text { with } \quad a_{\ell}\left(u_{\ell}, w_{\ell}\right)=f\left(w_{\ell}\right) \quad \text { for all } w_{\ell} \in V_{\ell} .
$$

We assume that also (2.2) is solvable and that the error estimate

$$
\left\|u-u_{\ell}\right\|_{0} \leq C_{e} h_{\ell}^{2 m}\|u\|_{2}
$$


holds, cf. Braess [4, p. 102], Hackbusch [13, (8.4.15b)]. Here, $2 m$ is the order of the differential operator, i.e., $\mathcal{H}^{1}$ is a subspace of $H^{m}(\Omega)$. As usual, $h_{\ell}$ is the size of the finite element mesh of $V_{\ell}$.

Together with the regularity assumption $\|u\|_{2} \leq C\|f\|_{0}$ from above, we obtain

$$
\left\|u-u_{\ell}\right\|_{0} \leq C_{0} h_{\ell}^{2 m}\|f\|_{0} .
$$

\subsection{Matrix Representation}

Let $\left\{b_{\ell, i}: i \in I_{\ell}\right\}$ be a basis of $V_{\ell}$, where $I_{\ell}$ is the corresponding index set (e.g., the set of nodal points). The coefficient vector space $\mathbb{R}^{I_{\ell}}$ is denoted by $\mathcal{U}_{\ell}$. The vectors in $\mathcal{U}_{\ell}$ are $\underline{u}_{\ell}=\left(u_{\ell, i}\right)_{i \in I_{\ell}}$, and $\mathcal{U}_{\ell}$ will be equipped with the usual Euclidean norm $\|\cdot\|_{\mathcal{U}_{\ell}}$ (scaled by a suitable factor to ensure (2.6) below), so that the adjoint mappings are given by the transposed matrices (maybe up to a fixed factor).

The isomorphism between $\mathcal{U}_{\ell}$ and $V_{\ell}$ is denoted by $\phi_{\ell}$ :

$$
\phi_{\ell}: \mathcal{U}_{\ell} \rightarrow V_{\ell} \text { with } u_{\ell}=\phi_{\ell} \underline{u}_{\ell}:=\sum_{i \in I_{\ell}} u_{\ell, i} b_{\ell, i} .
$$

The finite element matrix $A_{\ell}$ corresponding to $a_{\ell}(\cdot, \cdot)$ has the coefficients $a_{\ell, i j}=$ $a_{\ell}\left(b_{\ell, j}, b_{\ell, i}\right)$. The variational problem (2.2) is equivalent to

$$
A_{\ell} \underline{u}_{\ell}=\underline{f}_{\ell}
$$

with

$$
\underline{f}_{\ell}=\phi_{\ell}^{*} f, \quad \text { i.e., } \quad f_{\ell, i}=f\left(b_{\ell, i}\right)=\left(f, b_{\ell, i}\right)_{0} .
$$

As mentioned above, after a suitable scaling we require the equivalence of the Euclidean norm $\|\cdot\|_{\mathcal{U}_{\ell}}$ and the $\mathcal{H}^{0}$-norm:

$$
\frac{1}{C_{\phi^{-1}}}\left\|\underline{v}_{\ell}\right\|_{\mathcal{U}_{\ell}} \leq\left\|v_{\ell}\right\|_{0} \leq C_{\phi}\left\|\underline{v}_{\ell}\right\|_{\mathcal{U}_{\ell}} \quad \text { for all } v_{\ell}=\phi_{\ell} \underline{v}_{\ell} .
$$

\subsection{General Concept for the Multigrid Prolongation}

Main ingredients of the multigrid algorithm are the prolongation

$$
p: \mathcal{U}_{\ell-1} \rightarrow \mathcal{U}_{\ell}
$$

and the restriction $r=p^{*}: \mathcal{U}_{\ell} \rightarrow \mathcal{U}_{\ell-1}$. 
In the case of a conforming finite element discretisation with a finite element hierarchy $V_{0} \subset \ldots \subset V_{\ell-1} \subset V_{\ell}$, one obtains the following commutative diagram:

$$
\begin{aligned}
& V_{\ell-1} \underset{\iota}{\stackrel{\text { inclusion }}{\longrightarrow}} V_{\ell} \\
& \phi_{\ell-1} \uparrow \quad \uparrow \phi_{\ell} \\
& \mathcal{U}_{\ell-1} \underset{p}{\longrightarrow} \mathcal{U}_{\ell}
\end{aligned}
$$

In this case, the canonical prolongation is given by $p=\phi_{\ell}^{-1} \circ \phi_{\ell-1}$.

In the following we will admit $V_{\ell-1} \not \subset V_{\ell}$, and the inclusion is to be replaced by a suitable mapping

$$
\iota: V_{\ell-1} \rightarrow V_{\ell}
$$

Once $\iota$ has been given, we are able to define the prolongation and restriction by

$$
p=\phi_{\ell}^{-1} \circ \iota \circ \phi_{\ell-1} \quad \text { and } \quad r=p^{*}=\phi_{\ell-1}^{*} \circ \iota^{*} \circ\left(\phi_{\ell}^{*}\right)^{-1} \text {. }
$$

In the next section, we will propose a general construction of $\iota$ leading to the approximation property

$$
\left\|A_{\ell}^{-1}-p A_{\ell-1}^{-1} r\right\|_{\mathcal{U}_{\ell} \leftarrow \mathcal{U}_{\ell}} \leq C_{A} h_{\ell}^{2 m}
$$

which is an essential sufficient condition for the multigrid convergence (cf. Hackbusch $[12, \S 6.1 .3])$.

\section{Construction of the Prolongation}

\subsection{Spaces $\Sigma$ and $S$}

Although the algorithm needs only the mapping $\iota: V_{\ell-1} \rightarrow V_{\ell}$ (cf. (2.7)), the theoretical consideration will lead to a variational problem (4.7) on the sum $V_{\ell-1}+V_{\ell}$ and require $\iota$ to be defined and bounded on $V_{\ell-1}+V_{\ell}$ (or on a larger space). Since $\iota: V_{\ell} \rightarrow V_{\ell}$ has to be the identity, we must construct a bounded mapping $\iota: V_{\ell-1} \rightarrow V_{\ell}$ such that its restriction to $V_{\ell-1} \cap V_{\ell}$ is the identity.

In order to make the metric structure of the sum more transparent we will refer to a (possibly larger) space $\Sigma$ with

$$
V_{\ell-1}+V_{\ell} \subset \Sigma \subset \mathcal{H}^{0}
$$


The space $\Sigma$ and the space $S$ defined below belong to the index pair $(\ell-1, \ell)$, and $\Sigma_{\ell-1, \ell}$ and $S_{\ell-1, \ell}$ would be a more precise notation. For the sake of simplicity, we omit these indices.

Here we also note that the sum $V_{\ell-1}+V_{\ell}$ plays an important role when Stevenson [14] considers an axiomatic framework for the Cascadic multigrid algorithms suitable for nonconforming elements.

Next, we need an auxiliary space $S$, which is connected with $\Sigma$ and $V_{\ell}$ via the mappings $\sigma$ and $\pi$, as shown in the following commutative diagram:

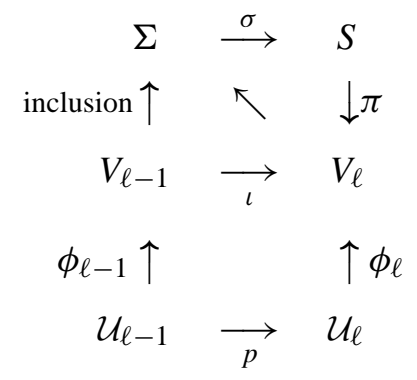

The desired mapping $\iota$ (more precisely, its extension to $\Sigma$ ) is the product

$$
\iota=\pi \circ \sigma: \Sigma \rightarrow V_{\ell}
$$

Before we will discuss the characteristic requirements concerning $\pi, \sigma$, and $\iota$ in the next subsection, for elucidating the formalism, we specify the spaces and mappings for the Crouzeix-Raviart element, i.e., for the simplest nonconforming finite elements (cf. Braess-Verfürth [5]).

Example 1. Let $\mathcal{T}_{\ell-1}$ be the coarse triangulation of the domain $\Omega$, while $\mathcal{T}_{\ell}$ is obtained by regular halving of all triangle sides. $V_{\ell}$ is the space of all piecewise linear functions which are continuous at the midpoints of edges in $\mathcal{T}_{\ell}$. Define the nodal point set $\mathcal{N}_{\ell}$ by all midpoints of edges in $\mathcal{T}_{\ell}$ (except boundary points in the case of Dirichlet conditions). For all $\alpha \in \mathcal{N}_{\ell}$, basis functions $b_{\ell, \alpha} \in V_{\ell}$ are defined by $b_{\ell, \alpha}(\beta)=\delta_{\alpha \beta}\left(\alpha, \beta \in \mathcal{N}_{\ell}\right)$ with the Kronecker symbol $\delta$. Then, $\mathcal{U}_{\ell}:=\ell_{2}\left(\mathcal{N}_{\ell}\right)$ is the coefficient space which is mapped by $\phi_{\ell}:\left(c_{\ell, \alpha}\right)_{\alpha \in \mathcal{N}_{\ell}} \mapsto u_{\ell}=\sum_{\alpha \in \mathcal{N}_{\ell}} c_{\ell, \alpha} b_{\ell, \alpha}$ onto $V_{\ell}$. Similarly, $V_{\ell-1}, \mathcal{U}_{\ell-1}$, and the isomorphism $\phi_{\ell-1}$ are defined.

An appropriate space $\Sigma$ is the space of piecewise linear elements with respect to the fine triangulation $\mathcal{T}_{\ell}$ that may be discontinuous at the edges of this triangulation. Obviously, $V_{\ell-1}+V_{\ell} \subset \Sigma$.

We set $S:=\mathcal{U}_{\ell}, \pi:=\phi_{\ell}$, and define $\sigma$ as follows: Every nodal point $\alpha \in \mathcal{N}_{\ell}$ is the 
midpoint of the common side of adjacent triangles $T$ and $T^{\prime}$ from $\mathcal{T}_{\ell}$. For $\alpha \in \mathcal{N}_{\ell}$ we set

$$
(\sigma v)_{\alpha}:=\frac{1}{2}\left[\left.v\right|_{T}(\alpha)+\left.v\right|_{T^{\prime}}(\alpha)\right] .
$$

Here, the linear function $\left.v\right|_{T}$ is understood to be extended to $\bar{T}$.

\subsection{Conditions on $\pi$ and $\sigma$}

The space $\Sigma$ will be equipped with the norm and the scalar product of $\mathcal{H}^{0}$, and $S$ is assumed to become a Hilbert space by a norm $\|\cdot\|_{S}$ and a scalar product $(\cdot, \cdot)_{S}$ whose specification may depend on the specific finite element space. The mapping $\sigma: \Sigma \rightarrow S$ is assumed to be bounded:

$$
\|\sigma\|_{S \leftarrow \mathcal{H}^{0}} \leq C_{\sigma} .
$$

Furthermore, $\pi: S \rightarrow V_{\ell}$ is required to be injective and bounded:

$$
\pi \text { is injective and }\|\pi\|_{V_{\ell} \leftarrow S} \leq C_{\pi} \text {. }
$$

The product $\iota=\pi \circ \sigma$ from (3.1) is assumed to be a projection onto $V_{\ell}$, i.e.,

$$
\left.\iota\right|_{V_{\ell}}=\left.\pi \circ \sigma\right|_{V_{\ell}}=i d: V_{\ell} \rightarrow V_{\ell} .
$$

Remark 3.2. If the conditions (3.2)-(3.4) hold, then $\iota: \Sigma \rightarrow V_{\ell} \subset \Sigma$ is a bounded projection onto $V_{\ell}$ :

$$
\|\iota\|_{\mathcal{H}^{0} \leftarrow \mathcal{H}^{0}} \leq C_{\iota}:=C_{\pi} C_{\sigma} .
$$

Moreover $\pi: S \rightarrow V_{\ell}$ is an isomorphism.

Proof: The boundedness (3.5) is a direct consequence of (3.2)-(3.3).

Since the range of $\pi$ is $V_{\ell}, \pi$ is injective and surjective. Hence, $\pi^{-1}=\left.\sigma\right|_{V_{\ell}}$, and $\pi$ is an isomorphism.

The introduction of the intermediate space $S$ gives us more freedom in the construction of the mappings. Of course, in many cases the set $S$ will coincide with $V_{\ell}$ or $\mathcal{U}_{\ell}$. We emphasise that only boundedness in $\mathcal{H}^{0}$ is required for $\iota$, while the concept of Brenner $[8,15]$ also refers to conditions with respect to the energy norm. 


\section{Coarse-Grid Correction and Approximation Property}

\subsection{Coarse-Grid Correction}

Let an approximation $\tilde{u}_{\ell}$ be given. Its defect $d_{\ell} \in\left(V_{\ell}\right)^{\prime}=V_{\ell}$ is defined by

$$
\left(d_{\ell}, w_{\ell}\right)_{0}=a_{\ell}\left(\tilde{u}_{\ell}, w_{\ell}\right)-f\left(w_{\ell}\right) \quad \text { for all } w_{\ell} \in V_{\ell} .
$$

Using (2.2) and the error

$$
e_{\ell}:=\tilde{u}_{\ell}-u_{\ell},
$$

one obtains a characterisation of $d_{\ell}$ by

$$
\left(d_{\ell}, w_{\ell}\right)_{0}=a_{\ell}\left(e_{\ell}, w_{\ell}\right) \quad \text { for all } w_{\ell} \in V_{\ell} .
$$

The residue of the linear system is $\underline{d}_{\ell}=A_{\ell} \underline{e}_{\ell}$ with $e_{\ell}=\phi_{\ell} \underline{e}_{\ell}$. Because of $\left(A_{\ell} \underline{e}_{\ell}, \underline{w}_{\ell}\right)_{\mathcal{U}_{\ell}}=a_{\ell}\left(e_{\ell}, w_{\ell}\right)$ with $w_{\ell}=\phi_{\ell} \underline{w}_{\ell}$ and $\left(d_{\ell}, w_{\ell}\right)_{0}=\left(d_{\ell}, \phi_{\ell} \underline{w}_{\ell}\right)_{0}=$ $\left(\phi_{\ell}^{*} \bar{d}_{\ell}, \underline{w}_{\ell}\right)_{\mathcal{u}_{\ell}}$, we conclude

Remark 4.1. The residue

$$
\underline{d}_{\ell}=A_{\ell} \underline{e}_{\ell} \in \mathcal{U}_{\ell}
$$

has the representation $\underline{d}_{\ell}=\phi_{\ell}^{*} d_{\ell}$ with $d_{\ell}$ being defined in (4.2).

The coarse-grid correction $e_{\ell-1} \in V_{\ell-1}$ approximates the finite element function $e_{\ell} \in V_{\ell}$. It is determined as the solution of the coarse-grid equation

$$
a_{\ell-1}\left(e_{\ell-1}, w_{\ell-1}\right)=a_{\ell}\left(e_{\ell}, \iota w_{\ell-1}\right) \quad \text { for all } w_{\ell-1} \in V_{\ell-1} .
$$

Here $\iota$ is the mapping specified in the previous section. Note that it is required for converting the function $w_{\ell-1}$ from $V_{\ell-1}$ into an element of $V_{\ell}$. The correction yields the new approximation $u_{\ell}^{\text {new }}:=\tilde{u}_{\ell}-\iota e_{\ell-1}$, cf. $[5,8]$. The error after the coarse-grid correction is obviously

$$
e_{\ell}^{n e w}=u_{\ell}^{n e w}-u_{\ell}=e_{\ell}-\iota e_{\ell-1} .
$$

\subsection{An Auxiliary Problem}

We will estimate $\left\|e_{\ell}^{n e w}\right\|_{0}$ by constructing an auxiliary problem for which $e_{\ell-1}$ and $e_{\ell}$ are the finite element solutions at the levels $\ell-1$ and $\ell$, respectively. To this end we introduce two Riesz representations of the residue.

Given $e_{\ell}$, define $\bar{r}_{\ell} \in S$ by

$$
\left(\bar{r}_{\ell}, \bar{w}\right)_{S}=a_{\ell}\left(e_{\ell}, \pi \bar{w}\right) \quad \text { for all } \bar{w} \in S .
$$


Similarly, let $g \in \Sigma$ be the solution of

$$
(g, s)_{0}=\left(\bar{r}_{\ell}, \sigma s\right)_{S} \quad \text { for all } s \in \Sigma .
$$

Lemma 4.2. $g$ has the representation $g=\sigma^{*} \bar{r}_{\ell}=\iota^{*}\left(\phi_{\ell}^{*}\right)^{-1} \underline{d}_{\ell}$ and satisfies

$$
\|g\|_{0} \leq C_{\sigma}\left\|\bar{r}_{\ell}\right\|_{S} \leq C_{\sigma} C_{\phi^{-1}} C_{\pi}\left\|\underline{d}_{\ell}\right\|_{\mathcal{U}_{\ell}} .
$$

Proof: First, we have $\bar{r}_{\ell}=\pi^{*}\left(\phi_{\ell}^{*}\right)^{-1} \underline{d}_{\ell}$ since $\left(\pi^{*}\left(\phi_{\ell}^{*}\right)^{-1} \underline{d}_{\ell}, \bar{w}\right)_{S}=\left(\pi^{*} d_{\ell}, \bar{w}\right)_{S}=$ $\left(d_{\ell}, \pi \bar{w}\right)_{0}=a_{\ell}\left(e_{\ell}, \pi \bar{w}\right)$ for all $\bar{w} \in S$. Combining this with $g=\sigma^{*} \bar{r}_{\ell}$ and $\iota^{*}=\sigma^{*} \pi^{*}$ we obtain the required representation of $g$.

Moreover, the inequality

$$
\begin{aligned}
\left\|\bar{r}_{\ell}\right\|_{S}^{2} & =\left(\bar{r}_{\ell}, \bar{r}_{\ell}\right)_{S} \underset{(4.6)}{=} a_{\ell}\left(e_{\ell}, \pi \bar{r}_{\ell}\right) \underset{(4.2)}{=} \\
& =\left(d_{\ell}, \pi \bar{r}_{\ell}\right)_{0} \underset{\operatorname{Remark} 4.1}{=}\left(\left(\phi_{\ell}^{*}\right)^{-1} \underline{d}_{\ell}, \pi \bar{r}_{\ell}\right)_{0}=\left(\underline{d}_{\ell}, \phi_{\ell}^{-1} \pi \bar{r}_{\ell}\right)_{\mathcal{U}_{\ell}} \\
& \leq\left\|\underline{d}_{\ell}\right\|_{\mathcal{U}_{\ell}}\left\|\phi_{\ell}^{-1} \pi \bar{r}_{\ell}\right\|_{\mathcal{U}_{\ell}} \leq\left\|\underline{d}_{\ell}\right\|_{\mathcal{U}_{\ell}} C_{\phi^{-1}} C_{\pi}\left\|\bar{r}_{\ell}\right\|_{S}
\end{aligned}
$$

yields $\left\|\bar{r}_{\ell}\right\|_{S} \leq C_{\phi^{-1}} C_{\pi}\left\|_{d_{\ell}}\right\|_{\mathcal{U}_{\ell}}$. Next we estimate $\|g\|_{0}^{2}=(g, g)_{0}=\left(\bar{r}_{\ell}, \sigma g\right)_{S} \leq$ $\left\|\bar{r}_{\ell}\right\|_{S}\|\sigma g\|_{S} \leq\left\|\bar{r}_{\ell}\right\|_{S} C_{\sigma}\|g\|_{0}$. After dividing by $\|g\|_{0}$ and inserting the estimate of $\bar{r}_{\ell}$ above, we obtain the required inequality.

Although the mapping $\iota$ need only be defined on $V_{\ell-1}$ for the computations, we have extended it to $V_{\ell-1}+V_{\ell}$. The aim of that process is an interesting property of $g$ which is the subject of

Proposition 4.3. The variational problem in $\mathcal{H}^{1}$,

$$
a(z, w)=(g, w)_{0} \quad \text { for all } w \in \mathcal{H}^{1},
$$

has the finite element solutions $e_{\ell-1}$ and $e_{\ell}$ from (4.4) and (4.1) at the levels $\ell-1$ and $\ell$, respectively.

Proof: a) On level $\ell$, we conclude from (3.4), (4.6), and (4.7) that

$$
a_{\ell}\left(e_{\ell}, w_{\ell}\right)=a_{\ell}\left(e_{\ell}, \pi \sigma w_{\ell}\right)=\left(\bar{r}_{\ell}, \sigma w_{\ell}\right)_{S}=\left(g, w_{\ell}\right)_{0} \quad \text { for all } w_{\ell} \in V_{\ell} .
$$

b) On level $\ell-1$, it follows from (4.4) and (3.1) that we have for all $w_{\ell-1} \in V_{\ell-1}$

$$
a_{\ell-1}\left(e_{\ell-1}, w_{\ell-1}\right)=a_{\ell}\left(e_{\ell}, \iota w_{\ell-1}\right)=a_{\ell}\left(e_{\ell}, \pi \sigma w_{\ell-1}\right)=\left(g, w_{\ell-1}\right)_{0} .
$$

The last equality was obtained as in part a) by (4.6) and (4.7). 


\subsection{Approximation Property}

Let $z \in \mathcal{H}^{1}$ be the solution of the variational problem in Proposition 4.3. Because of $g \in \mathcal{H}^{0}$ and the regularity condition in Section $2.1, z$ belongs to $\mathcal{H}^{2}$. The error estimate (2.4) yields the statement that $z$ and its finite element approximations $e_{\ell-1}$ and $e_{\ell}$ satisfy

$$
\left\|z-e_{j}\right\|_{0} \leq C_{0} h_{j}^{2 m}\|g\|_{0} \quad \text { for } j=\ell-1, \ell .
$$

The error $e_{\ell}^{\text {new }}=\phi_{\ell} \underline{e}_{\ell}^{\text {new }}$ from (4.5) after the coarse-grid correction will be estimated in the following proposition. Here, we make use of the standard assumption (on the mesh size ratio)

$$
h_{\ell-1} \leq C_{h} h_{\ell}
$$

which usually holds with $C_{h}=2$.

Proposition 4.4. Under the previous assumptions, the estimate

$$
\left\|\underline{e}_{\ell}^{n e w}\right\|_{\mathcal{U}_{\ell}} \leq C_{A} h_{\ell}^{2 m}\left\|\underline{d}_{\ell}\right\|_{\mathcal{U}_{\ell}}
$$

holds for all $\underline{d}_{\ell} \in \mathcal{U}_{\ell}$ with $C_{A}:=C_{\phi^{-1}}^{2} C_{\iota} C_{0}\left(1+C_{h}^{2 m}\right) C_{\sigma} C_{\pi}$.

Proof: $\left\|\underline{e}_{\ell}^{\text {new }}\right\|_{\mathcal{U}_{\ell}} \leq C_{\phi^{-1}}\left\|e_{\ell}^{n e w}\right\|_{0}$ holds because of (2.6). From (3.4) and (3.5) it follows that

$$
\left\|e_{\ell}^{n e w}\right\|_{0}=\left\|e_{\ell}-\iota e_{\ell-1}\right\|_{0}=\left\|\iota\left(e_{\ell}-e_{\ell-1}\right)\right\|_{0} \leq C_{\iota}\left\|e_{\ell}-e_{\ell-1}\right\|_{0} .
$$

Now (4.9) implies

$$
\left\|e_{\ell}-e_{\ell-1}\right\|_{0} \leq\left\|e_{\ell}-z\right\|_{0}+\left\|z-e_{\ell-1}\right\|_{0} \leq C_{0}\left(h_{\ell}^{2 m}+h_{\ell-1}^{2 m}\right)\|g\|_{0} .
$$

Moreover, $h_{\ell}^{2 m}+h_{\ell-1}^{2 m} \leq\left(1+C_{h}^{2 m}\right) h_{\ell}^{2 m}$.

Finally, we insert (4.8) to obtain

$$
\left\|e_{\ell}-e_{\ell-1}\right\|_{0} \leq C_{e e} h_{\ell}^{2 m}\left\|\underline{d}_{\ell}\right\|_{\mathcal{U}_{\ell}}
$$

with $C_{e e}:=C_{0} C_{\sigma} C_{\phi^{-1}} C_{\pi}\left(1+C_{h}^{2 m}\right)$. After inserting this estimate into (4.11) the proof is complete.

In order to derive the desired approximation property (2.9) from the error estimate, we return to the vector representation of the coarse-grid correction

$$
\underline{e}_{\ell-1}=A_{\ell-1}^{-1} \phi_{\ell-1}^{*} g=A_{\ell-1}^{-1} \phi_{\ell-1}^{*} \sigma^{*} \pi^{*}\left(\phi_{\ell}^{*}\right)^{-1} \underline{d}_{\ell}=A_{\ell-1}^{-1} \phi_{\ell-1}^{*} \iota^{*}\left(\phi_{\ell}^{*}\right)^{-1} \underline{d}_{\ell}
$$


(cf. Lemma 4.2). Therefore, the representation of $\underline{e}_{\ell}^{\text {new }}$ is

$$
\begin{aligned}
\underline{e}_{\ell}^{\text {new }} & =\phi_{\ell}^{-1} e_{\ell}^{n e w}=\phi_{\ell}^{-1}\left(e_{\ell}-\iota e_{\ell-1}\right)=\underline{e}_{\ell}-\phi_{\ell}^{-1} \iota \phi_{\ell-1} \underline{e}_{\ell-1} \\
& =A_{\ell}^{-1} \underline{d}_{\ell}-\phi_{\ell}^{-1} \iota \phi_{\ell-1} A_{\ell-1}^{-1} \phi_{\ell-1}^{*} \iota^{*}\left(\phi_{\ell}^{*}\right)^{-1} \underline{d}_{\ell} \\
& =\left(A_{\ell}^{-1}-p A_{\ell-1}^{-1} r\right) \underline{d}_{\ell}
\end{aligned}
$$

with $p$ and $r$ from (2.8). The inequality (4.10) is equivalent to (2.9). This proves

Proposition 4.5. Under the required assumptions, the approximation property (2.9) holds with the constant $C_{A}:=C_{\phi^{-1}}^{2} C_{\iota} C_{0}\left(1+C_{h}^{2 m}\right) C_{\sigma} C_{\pi}$.

Finally we note that the approximation property for the framework in [4, p. 222] is obtained from Proposition 4.4 and (4.1),

$$
\left\|e_{\ell}-\iota e_{\ell-1}\right\|_{0} \leq C_{A} h_{\ell}^{2 m}\left\|A_{\ell} \underline{e}_{\ell}\right\|_{\mathcal{U}_{\ell}} .
$$

\section{Smoothing Property and Multigrid Convergence}

\subsection{Smoothing Property}

It is well known that the convergence of multigrid algorithms can only be proved if there is an inverse property which fits to the error estimates in Section 2.2. Specifically, we assume that the matrix $A_{\ell}$ is bounded by

$$
\left\|A_{\ell}\right\|_{\mathcal{U}_{\ell} \leftarrow \mathcal{U}_{\ell}} \leq C_{B} h_{\ell}^{-2 m} .
$$

If, in addition, $A_{\ell}$ is positive definite, the simplest possible iteration (the Richardson iteration) is already a smoothing iteration:

$$
\begin{aligned}
u_{\ell} \mapsto \mathcal{S}_{\ell}\left(u_{\ell}, f_{\ell}\right) & :=u_{\ell}-C_{B}^{-1} h_{\ell}^{2 m}\left(A_{\ell} u_{\ell}-f_{\ell}\right), \\
\mathcal{S}_{\ell} & :=I-C_{B}^{-1} h_{\ell}^{2 m} A_{\ell},
\end{aligned}
$$

since it satisfies the smoothing property

$$
\left\|A_{\ell} \mathcal{S}_{\ell}^{v}\right\|_{\mathcal{U}_{\ell} \leftarrow \mathcal{U}_{\ell}} \leq \eta(v) h_{\ell}^{-2 m} \quad \text { for all } v \geq 0
$$

with $\eta(v):=C_{B} \eta_{0}(v)$ and $\eta_{0}(v):=v^{v} /(v+1)^{(v+1)}$ (cf. [12, §6.2]). The cases of $A_{\ell}$ not being positive definite or of other smoothing iterations are described in [12], too.

In addition, we assume that $\mathcal{S}_{\ell}^{v}$ remains bounded:

$$
\left\|\mathcal{S}_{\ell}^{\nu}\right\|_{\mathcal{U}_{\ell} \leftarrow \mathcal{U}_{\ell}} \leq C_{S} \quad \text { for all } v \geq 0, \ell \geq 1
$$


Under the previous assumptions, the Richardson iteration satisfies (5.3) with $C_{S}=$ 1.

\subsection{Multigrid Convergence}

The iteration matrix of the two-grid iteration (with pre-smoothing, only) equals

$$
M_{\ell}^{T G M}(v)=\left(A_{\ell}^{-1}-p A_{\ell-1}^{-1} r\right)\left(A_{\ell} \mathcal{S}_{\ell}^{\nu}\right),
$$

where $v$ is the number of smoothing iterations described by the iteration matrix $\mathcal{S}_{\ell}$. The approximation property (2.9) and the smoothing property (5.2) yield

$$
\left\|M_{\ell}^{T G M}(v)\right\|_{\mathcal{U}_{\ell} \leftarrow \mathcal{U}_{\ell}} \leq C_{A} \eta(v) \quad \text { for all } v \geq 0 .
$$

Let $\zeta<1$ be given. Since $\lim _{v \rightarrow \infty} \eta(v)=0$, the right-hand side is bounded by $C_{A} \eta(v) \leq \zeta<1$ for all $v \geq \underline{v}(\zeta)$, where $\underline{v}(\zeta)$ is independent of the mesh size $h_{\ell}$. In the following, the number $v$ is fixed.

Replacing the exact solution of the coarse-grid problem by two iterations of the multigrid method, we obtain the W-cycle. Its iteration matrix $M_{\ell}^{M G M}=M_{\ell}^{M G M}(v)$ is given by the recursion

$$
\begin{aligned}
& M_{1}^{M G M}=M_{1}^{T G M}, \\
& M_{\ell}^{M G M}=M_{\ell}^{T G M}+p\left(M_{\ell-1}^{M G M}\right)^{2} A_{\ell-1}^{-1} r A_{\ell} \mathcal{S}_{\ell}^{v} \quad \text { for } \ell \geq 2 .
\end{aligned}
$$

Let $\zeta_{\ell}:=\left\|M_{\ell}^{M G M}\right\|_{\mathcal{U}_{\ell} \leftarrow \mathcal{U}_{\ell}}$. From the two-grid analysis we know $\zeta_{1} \leq \zeta<1$. Equation (5.4) together with the estimates $\|p\|_{\mathcal{U}_{\ell} \leftarrow \mathcal{U}_{\ell-1}} \leq C_{\phi} C_{l} C_{\phi^{-1}}=$ : $C_{p}$ (cf. (2.8)) yields the recursive inequality

$$
\zeta_{\ell} \leq \zeta+C_{p} \zeta_{\ell-1}^{2}\left\|A_{\ell-1}^{-1} r A_{\ell} \mathcal{S}_{\ell}^{v}\right\|_{\mathcal{U}_{\ell-1} \leftarrow \mathcal{U}_{\ell}} .
$$

The essential step is to prove

$$
\left\|A_{\ell-1}^{-1} r A_{\ell} \mathcal{S}_{\ell}^{v}\right\|_{\mathcal{U}_{\ell-1} \leftarrow \mathcal{U}_{\ell}} \leq C,
$$

since then (5.5) implies $\zeta_{\ell} \leq \zeta+C^{*} \zeta_{\ell-1}^{2}$. Note that we have $\zeta<1 / 4 C^{*}$ if the number of smoothing steps $v$ is sufficiently large. Now the recursion relation implies $\zeta_{\ell} \leq 2 \zeta /\left(1+\sqrt{1-4 \zeta C^{*}}\right)$ (cf. [12, (7.1.7e)]). This proves mesh independent convergence rates for sufficiently many smoothing steps $v$.

In [12, Lemma 7.1.5], the estimate (5.6) is established under the condition $\left\|\underline{u}_{\ell-1}\right\|_{\mathcal{U}_{\ell-1}} \leq \underline{C}_{p}\left\|p \underline{u}_{\ell-1}\right\|_{\mathcal{U}_{\ell}}$. Usually, this inequality is valid, but here it is not possible to prove this estimate via assumptions on $\sigma$, since, typically, $\sigma$ is not injective 
on $\Sigma$. Therefore, we provide a different proof. Using $\left\|A_{\ell-1}^{-1} r A_{\ell} \mathcal{S}_{\ell}^{v}\right\|_{\mathcal{U}_{\ell-1} \leftarrow \mathcal{U}_{\ell}} \leq$ $C_{S}\left\|A_{\ell-1}^{-1} r A_{\ell}\right\|_{\mathcal{U}_{\ell-1} \leftarrow \mathcal{U}_{\ell}}$ (cf. (5.3)), it remains to estimate $A_{\ell-1}^{-1} r A_{\ell}$.

Equation (4.13) can be rewritten as $\underline{e}_{\ell-1}=A_{\ell-1}^{-1} r \underline{d}_{\ell}=A_{\ell-1}^{-1} r A_{\ell} \underline{e}_{\ell}$. Combining (4.12) with $\underline{d}_{\ell}=A_{\ell} \underline{e}_{\ell}$ and (5.1), we infer $\left\|e_{\ell}-e_{\ell-1}\right\|_{0} \leq C_{e \ell} C_{B}\left\|\underline{e}_{\ell}\right\|_{\mathcal{U}_{\ell}}$. This shows

$$
\left\|e_{\ell-1}\right\|_{0} \leq\left\|e_{\ell-1}-e_{\ell}\right\|_{0}+\left\|e_{\ell}\right\|_{0} \leq\left(C_{e e} C_{B}+C_{\phi}\right)\left\|\underline{e}_{\ell}\right\|_{\mathcal{U}_{\ell}}
$$

and $\left\|\underline{e}_{\ell-1}\right\|_{\mathcal{U}_{\ell-1}} \leq C_{\phi^{-1}}\left(C_{e \ell} C_{B}+C_{\phi}\right)\left\|_{\underline{e}_{\ell}}\right\|_{\mathcal{U}_{\ell}}$. Since this inequality holds for all $\underline{e}_{\ell} \in \mathcal{U}_{\ell}$, the representation $\underline{e}_{\ell-1}=A_{\ell-1}^{-1} r A_{\ell} \underline{e}_{\ell}$ yields the next lemma.

Lemma 5.1. Under the previous conditions, $\left\|A_{\ell-1}^{-1} r A_{\ell}\right\|_{\mathcal{U}_{\ell-1} \leftarrow \mathcal{U}_{\ell}} \leq C$ holds with $C:=C_{\phi^{-1}}\left(C_{e e} C_{B}+C_{\phi}\right)$.

Since Lemma 5.1 implies (5.6), the multigrid convergence is proved.

\section{Application to Non-Matching Grids}

In this section, we describe and analyse a multigrid method for solving systems of algebraic equations arising from a finite element method based on non-matching triangulations. The discretisation is done by the mortar technique, see [2, 3]. A multigrid method is presented and analysed which makes use of the general scheme presented in the previous sections.

\subsection{Discrete Problem}

For simplicity of presentation we restrict ourselves to the Poisson equation and assume that (2.1) is of the form: Find $u \in H_{0}^{1}(\Omega)$ such that

$$
a(u, v) \equiv \int_{\Omega} \nabla u \nabla v d x=f(v) \equiv \int_{\Omega} f v d x .
$$

Moreover, let $\Omega \subset \mathbb{R}^{2}$ be a polygonal domain and

$$
\bar{\Omega}=\sum_{j \in I} \bar{\Omega}_{j}
$$

where $\Omega_{j}$ are polygons. We also assume that $\Omega_{j}$ form a 'triangulation', i.e., $\bar{\Omega}_{i} \cap \bar{\Omega}_{j}$ for $i \neq j$ are empty or have a common edge or vertex. In the mortar method nomenclature, this case is called geometrically conforming. A more general case, which is called geometrically nonconforming, will be discussed in Section 7.

Let $\Gamma_{i j}$ be the open part of $\bar{\Omega}_{i} \cap \bar{\Omega}_{j}=\bar{\Gamma}_{i j}$. The union of the internal boundaries 
yields the skeleton

$$
\Gamma=\bigcup_{j \in I} \partial \Omega_{j} \backslash \partial \Omega
$$

Let $\mathcal{T}_{0, j}$ be a coarsest triangulation in $\Omega_{j}$ with the mesh size ${ }^{1} h_{0}$. The $\ell$-times refined triangulation is $\mathcal{T}_{\ell, j}$ with mesh parameter $h_{\ell}=h_{0} 2^{-\ell}$. The level number $\ell$ is assumed to range from 0 to $\ell_{j}$. Although, in general, $\ell_{j}$ may be different in different $\Omega_{j}$, we assume for simplicity that the number of levels in each $\Omega_{j}$ is the same, i.e., $\ell_{j}=\ell_{\max }$ for all $j \in I$. The standard finite element space of continuous and piecewise linear functions over the triangulation $\mathcal{T}_{\ell, j}$ is denoted by $S_{\ell, j}\left(\Omega_{j}\right)$. Let

$$
X_{\ell}:=X_{\ell}(\Omega):=\prod_{j \in I} S_{\ell, j}\left(\Omega_{j}\right)
$$

for $\ell=0, \ldots, \ell_{\max }$. Note that $X_{\ell} \not \subset H^{1}(\Omega)$ and functions from $X_{\ell}$ do not satisfy any continuity condition across the internal boundaries $\Gamma_{i j}$.

The nodal points of $X_{\ell}$ form the set

$$
\overline{\mathcal{N}}_{\ell}:=\bigcup_{j \in I} \overline{\mathcal{N}}_{\ell, j}
$$

where $\overline{\mathcal{N}}_{\ell, j}$ is the set of nodal points of $\mathcal{T}_{\ell, j}$ on $\bar{\Omega}_{j}$.

Remark 6.1. The precise notation of a nodal point $p \in \overline{\mathcal{N}}_{\ell}$ is to be made by the pair $^{2}\left(x_{p}, \Omega_{j(p)}\right)$. An evaluation of a function $u$ at $p$ means values of $\left.u\right|_{\Omega_{j(p)}}$ at $x_{p}$, i.e., the continuation of the function $u$ defined on $\Omega_{j(p)}$ to $x_{p} \in \bar{\Omega}_{j(p)}$. In particular, at a cross point there are several nodal points $\left(x_{p}, \Omega_{j(p)}\right)$ with identical position $x_{p}$ but different $\Omega_{j(p)}$.

\subsubsection{Mortar Spaces}

To define suitable spaces for discretisation of (6.1) we need to impose some constraints on the jumps of functions from $X_{\ell}(\Omega)$ on $\Gamma_{i j}$ which are called mortar

\footnotetext{
${ }_{1}^{1}$ Although the mortar method works with different mesh sizes $h_{\ell, j}$ in the different subdomains $\Omega_{j}$, we assume comparable sizes $h_{\ell}$ for all $j$. The reason is that the fast multigrid convergence requires similar mesh parameters.

${ }^{2}$ In total, we will have three index mappings. Here, $p \in \mathcal{N}_{\ell} \mapsto j(p) \in I$ maps the vertex into the index of the related subdomain. In Section 6.1, we shall introduce $m \in \mathcal{M} \mapsto i_{m} \in I$, where $m$ is the master index and $i_{m}$ the related subdomain index. Similarly, $m \in \mathcal{M} \mapsto j_{m} \in I$ maps into the subdomain index of the slave edge.
} 
conditions. For this purpose, decompose the skeleton (6.2) into

$$
\bar{\Gamma}=\bigcup_{m \in \mathcal{M}} \bar{\gamma}_{m}, \quad \gamma_{m} \neq \gamma_{n} \text { if } m \neq n, n, m \in \mathcal{M}
$$

With each master index $m \in \mathcal{M}$, we associate a pair $(i, j)=\left(i_{m}, j_{m}\right) \in I \times I$ such that $\gamma_{m}=\Gamma_{i j}$ is an open edge of $\Omega_{i}$ common to $\Omega_{j}$. It is called master (mortar) and values on $\gamma_{m}$ will be continued from the values in $\Omega_{i} . \Gamma_{j i}=\Gamma_{i j}$ as an open edge of $\Omega_{j}$ is denoted by $\delta_{m}$. It is called slave (nonmortar), and values on $\delta_{m}$ will be continued from the values in $\Omega_{j}$.

To be more specific, let $m \in \mathcal{M}, i=i_{m}, j=j_{m} \in I$ be as before. The (twodimensional) triangulation $\mathcal{T}_{\ell, i}, \ell \in\left\{0, \ldots, \ell_{\max }\right\}$, induces a (one-dimensional) mesh $\mathcal{T}_{\ell, i} \cap \bar{\gamma}_{m}$ on $\gamma_{m}$. Similarly, one considers the mesh $\mathcal{T}_{\ell, j} \cap \bar{\delta}_{m}$ on $\delta_{m}$. Usually, both meshes are different. The trace spaces

$$
S_{\ell, m, j_{m}}\left(\delta_{m}\right):=\left.S_{\ell, j_{m}}\left(\Omega_{j_{m}}\right)\right|_{\delta_{m}}
$$

are associated to these meshes on the interfaces. We note that the nodal points $p \in \overline{\mathcal{N}}_{\ell, i}$ and $q \in \overline{\mathcal{N}}_{\ell, j}$ are considered as different even if $x_{p} \in \mathcal{T}_{\ell, i} \cap \bar{\gamma}_{m}$ and $x_{q} \in \mathcal{T}_{\ell, j} \cap \bar{\delta}_{m}$ coincide since they are associated to different domains (see Remark 6.1 above).

By definition, $\gamma_{m}$ and $\delta_{m}$ are open sets. The boundary $\partial \gamma_{m}\left(\partial \delta_{m}\right)$ consists of the two endpoints of the edge $\gamma_{m}\left(\delta_{m}\right)$.

Next, we introduce the space $M_{\ell, m}\left(\delta_{m}\right)$ as a subspace of $S_{\ell, m, j_{m}}\left(\delta_{m}\right)$ consisting of piecewise linear continuous functions on $\mathcal{T}_{\ell, j_{m}} \cap \bar{\delta}_{m}$, which are constant on the elements intersecting $\partial \delta_{m}$ (i.e., on the two end intervals of the mesh $\mathcal{T}_{\ell, j_{m}} \cap \bar{\delta}_{m}$ ). We say that $v_{\ell} \in X_{\ell}(\Omega)$ satisfies the mortar condition for $m \in \mathcal{M}$ if

$$
\int_{\Gamma_{i_{m}, j_{m}}}\left(\left.v_{\ell, i_{m}}\right|_{\gamma_{m}}-\left.v_{\ell, j_{m}}\right|_{\delta_{m}}\right) \psi d s=0 \quad \text { for all } \psi \in M_{\ell, m}\left(\delta_{m}\right)
$$

The first factor in the integral is the jump of $v_{\ell}$ across $\Gamma_{i_{m}}, j_{m}$ and often denoted as $\left.\left[v_{\ell}\right]\right|_{\Gamma_{i_{m}, j_{m}}}$. Therefore the elements in $M_{\ell, m}\left(\delta_{m}\right)$ can be understood as Lagrange multipliers for the matching conditions.

We are now able to define the mortar space for discretising (6.1). Let

$V_{\ell}(\Omega):=\left\{v_{\ell} \in X_{\ell}(\Omega): v_{\ell}\right.$ satisfies the mortar condition (6.4) for each $\left.m \in \mathcal{M}\right\}$

The discrete problem is of the form: Find $u_{\ell} \in V_{\ell}(\Omega)$ such that

$$
a_{\ell}\left(u_{\ell}, v_{\ell}\right)=f\left(v_{\ell}\right) \quad \text { for all } v_{\ell} \in V_{\ell}(\Omega)
$$


(cf. (2.2)), where

$$
\begin{aligned}
a_{\ell}\left(u_{\ell}, v_{\ell}\right) & :=\sum_{j \in I} \int_{\Omega_{j}} \nabla u_{\ell, j} \nabla v_{\ell, j} d x, \\
f\left(v_{\ell}\right) & :=\sum_{j \in I} \int_{\Omega_{j}} f v_{\ell, j} d x .
\end{aligned}
$$

This problem has a unique solution and is stable. Moreover the error bound

$$
\left\|u-u_{\ell}\right\|_{L_{2}(\Omega)} \leq C h_{\ell}^{2}\|u\|_{H^{2}(\Omega)}
$$

holds, where $C$ is independent of $h_{\ell}$ and $u$. For the proof see [7, Theorem 4.1]. The error estimate is stated there for mortar elements that are continuous at the cross points. Fortunately, the bound of the consistency error in Theorem 4.1 and the estimate in Lemma 3.4 are independent of assumptions on cross points. Inequality (6.6) is required in (2.3). A proof of the error estimate in $H^{1}(\Omega)$ can be found in [2] and [3] and the $L_{2}$ estimate is also given in [1] without a proof.

Our goal is to design and analyse a multigrid method for solving the linear system (6.9) from below corresponding to (6.5). The next three subsections will be a preparation for that.

\subsubsection{Matrix Form}

We rewrite the problem (6.5) in a matrix form using basis functions of $V_{\ell}(\Omega)$, i.e., basis functions satisfying the mortar condition. We emphasise that we do not require continuity at the cross points for the functions in $V_{\ell}(\Omega)$.

Assume that $v_{\ell} \in X_{\ell}$ satisfies the mortar condition (6.4). Note that $\left.v_{\ell, j_{m}}\right|_{\delta_{m}}$ can be computed at interior nodal points of $\mathcal{T}_{\ell, j_{m}} \cap \delta_{m}$ from the values of $\left.v_{\ell, i_{m}}\right|_{\gamma_{m}}$ on $\bar{\gamma}_{m}$ and the two values of $v_{\ell, j_{m}}$ at the endpoints of $\delta_{m}$. The restriction of a function $v$ on $\delta_{m}$ to the endpoints of $\delta_{m}$ is denoted by $\operatorname{Tr}_{m} v$. We have $\left.v_{\ell, j_{m}}\right|_{\delta_{m}}:=$ $\Pi_{\ell, m}\left(v_{\ell, i_{m}}, T r_{m} v_{\ell, j_{m}}\right)$, where $\Pi_{\ell, m}$ is a mapping from $L_{2}\left(\Gamma_{i_{m}, j_{m}}\right) \times \operatorname{Tr}_{m}\left(\delta_{m}\right)$ onto $S_{\ell, m, j_{m}}\left(\delta_{m}\right)$ (see (6.3)) and given by the solution $w \in S_{\ell, m, j_{m}}\left(\delta_{m}\right)$ of

$$
\begin{aligned}
\int_{\Gamma_{i_{m}, j_{m}}}\left(w-v_{\ell, i_{m}}\right) \psi d s & =0 \quad \text { for all } \psi \in M_{\ell, m}\left(\delta_{m}\right), \\
\operatorname{Tr}_{m} w & =\operatorname{Tr}_{m} v_{\ell, j_{m}} .
\end{aligned}
$$

To find $\Pi_{\ell, m}\left(v_{\ell, i_{m}}, \operatorname{Tr}_{m} v_{\ell, j_{m}}\right)$, we only need to solve a system with a tridiagonal mass matrix.

Let $\mathcal{N}_{\ell, j}$ and $\mathcal{N}_{\ell}$ be the set of nodal points of $\overline{\mathcal{N}}_{\ell, j}$ and $\overline{\mathcal{N}}_{\ell}$, respectively, except 
those which belong to interiors of the slave edges, i.e.,

$$
\mathcal{N}_{\ell, j}:=\overline{\mathcal{N}}_{\ell, j} \backslash \bigcup_{m \in \mathcal{M}} \delta_{m}, \quad \mathcal{N}_{\ell}:=\bigcup_{j \in I} \mathcal{N}_{\ell, j}
$$

In the following, we describe the Lagrange basis $\left\{b_{\ell, p}: p \in \mathcal{N}_{\ell}\right\}$ of $V_{\ell}(\Omega)$. It is uniquely defined by $b_{\ell, p} \in V_{\ell}(\Omega)$ and $b_{\ell, p}\left(x_{q}\right)=\delta_{p q}$ (Kronecker symbol $\delta$ ). We discuss the structure of these basis functions in detail.

Recalling Remark 6.1, we associate with each $p=\left(x_{p}, \Omega_{j(p)}\right) \in \mathcal{N}_{\ell}$ a basis function $b_{\ell, p} \in V_{\ell}(\Omega)$ and distinguish three cases. For the standard nodal basis function in $X_{\ell}$ corresponding to the vertex $p \in \overline{\mathcal{N}}_{\ell, j(p)}$, the subdomain $\Omega_{j(p)}$, and the triangulation $\mathcal{T}_{\ell, j(p)}$, we use the notation $\varphi_{\ell, p}$. Concerning the notations $j(p)$, $i_{m}$ and $j_{m}$ we refer to Footnote 2 .

Case I (interior nodal point) For $x_{p} \in \Omega_{j(p)},\left.b_{\ell, p}\right|_{\Omega_{j(p)}}=\varphi_{\ell, p}$ is the standard nodal basis function on $\bar{\Omega}_{j(p)}$ and $b_{\ell, p}=0$ on $\Omega \backslash \bar{\Omega}_{j(p)}$. In this case, $b_{\ell, p}$ is continuous on the whole domain $\Omega$.

Case II (nodal point on interior of an interior boundary) Let $m \in \mathcal{M}$ and $x_{p} \in \gamma_{m}$. Hence, $i_{m}=j(p)$. Obviously, $p \in \mathcal{N}_{\ell, i_{m}}$, i.e., the nodal point lies in the interior of a master edge $\gamma_{m}$ of $\Omega_{i_{m}}$. Then

$$
b_{\ell, p}= \begin{cases}\varphi_{\ell, p} & \text { on } \bar{\Omega}_{i_{m}}, \\ \Pi_{\ell, m}\left(\varphi_{\ell, p}, 0\right) & \text { on } \bar{\delta}_{m} \subset \partial \Omega_{j_{m}}, \\ 0 & \text { at all other nodal points in } \bar{\Omega} \backslash\left(\bar{\Omega}_{i_{m}} \cup \bar{\delta}_{m}\right) .\end{cases}
$$

The support of $b_{\ell, p}$ consists of all triangles from $\mathcal{T}_{\ell, j_{m}}$ which touch the (open) slave edge $\delta_{m}$ and the triangles from $\mathcal{T}_{\ell, i_{m}}$ which touch $x_{p}$. Note that $b_{\ell, p}$ is discontinuous at $\Gamma_{i_{m}, j_{m}}$.

Case III (cross point as nodal point) A cross point may belong to several $\bar{\Omega}_{i}$ and to various $\partial \gamma_{m}$ or $\partial \delta_{m}$. Let $p=\left(x_{p}, \Omega_{j(p)}\right)$. The subdomain $\Omega_{j(p)}$ has two edges joining at $x_{p}$. Each of these edges may be mortar edges $\gamma_{m}$ (then $i_{m}=j(p)$ ) or nonmortar edges $\delta_{m}$ (then $\left.j_{m}=j(p)\right)$. We define $b_{\ell, p}$ by $b_{\ell, p}(p)=1$ and

$$
b_{\ell, p}= \begin{cases}\varphi_{\ell, p} & \text { on } \gamma_{m} \subset \partial \Omega_{i_{m}} \text { if } x_{p} \in \partial \gamma_{m}, i_{m}=j(p), \\ \Pi_{\ell, m}\left(0, T r_{m} \varphi_{\ell, p}\right) & \text { on } \delta_{m} \subset \partial \Omega_{i_{m}} \text { if } x_{p} \in \partial \delta_{m}, i_{m}=j(p), \\ \Pi_{\ell, m}(\varphi \ell, p, 0) & \text { on } \delta_{m} \subset \partial \Omega_{j_{m}} \text { if } x_{p} \in \partial \delta_{m}, j_{m}=j(p), \\ 0 & \text { at all other nodal points in } \bar{\Omega} .\end{cases}
$$

The first two rows correspond to mortar/nonmortar edges associated to the subdomain $\Omega_{j(p)}$, while in the third row $\delta_{m}$ is a nonmortar belonging to a neighbouring subdomain. 
Using the basis functions above, we have $V_{\ell}(\Omega)=\operatorname{span}\left\{b_{\ell, p}: p \in \mathcal{N}_{\ell}\right\}$. Problem (6.5) takes the matrix form

$$
A_{\ell} \underline{u}_{\ell}=\underline{f}_{\ell} .
$$

The matrix $A_{\ell}$ is symmetric and positive definite. Moreover it follows from the usual inverse estimates for $X_{\ell}$ and $V_{\ell} \subset X_{\ell}$ that the eigenvalues $\lambda_{i}\left(A_{\ell}\right)$ of $A_{\ell}$ satisfy the inequalities

$$
C_{0} \leq \lambda_{i}\left(A_{\ell}\right) \leq C_{1} h_{\ell}^{-2}
$$

where $C_{0}$ and $C_{1}$ are independent of $h_{\ell}$.

\subsubsection{Nonconformity}

In Section 6.1.2, we have constructed a family of finite element spaces $V_{\ell}(\Omega)$ $\left(\ell=0,1, \ldots, \ell_{\max }\right)$ which satisfy the mortar condition (6.4) of the respective level. We have started with nested spaces

$$
X_{0}(\Omega) \subset X_{1}(\Omega) \subset \ldots \subset X_{\ell}(\Omega) \subset \prod L_{2}\left(\Omega_{j}\right)
$$

However, the subspaces $V_{\ell}(\Omega)$ are not nested. The reason is that a function $\left.u_{\ell-1}\right|_{\Omega_{i_{m}}}=\left.u_{\ell}\right|_{\Omega_{i_{m}}}$ belonging to $S_{\ell-1, i_{m}}\left(\Omega_{i_{m}}\right) \subset S_{\ell, i_{m}}\left(\Omega_{i_{m}}\right)$ yields different values $u_{\ell-1}\left|\Omega_{j m} \neq u_{\ell}\right|_{\Omega_{j m}}$ on the slave side since the mortar condition for level $\ell-1$ does not imply the mortar condition for level $\ell$, in general. Hence,

$$
V_{0}(\Omega) \not \subset V_{1}(\Omega) \not \subset \ldots \not \subset V_{\ell}(\Omega) .
$$

\subsection{Estimates}

The analysis of the multigrid method reduces to the analysis of the two-grid one (cf. Subsection 5.2). Therefore the facts needed for that analysis are formulated for the levels $\ell$ and $\ell-1$.

\subsection{1. $L_{2}$-Stability of the Mortar Projection}

It is known that the mortar projection $\Pi_{\ell, m}$ (cf. (6.7) and (6.8)) is $L_{2}$-stable.

Lemma 6.2. Assume that $v_{\ell} \in X_{\ell}$ satisfies the mortar condition on $\delta_{m}=\gamma_{m}=$ $\Gamma_{i_{m}, j_{m}}$. If $\operatorname{Tr}_{m} v_{\ell, j_{m}}=0$, then

$$
\left\|v_{\ell, j_{m}}\right\|_{L_{2}\left(\delta_{m}\right)} \leq C\left\|v_{\ell, i_{m}}\right\|_{L_{2}\left(\gamma_{m}\right)}
$$

where the constant $C$ is independent of $h_{\ell}$. 
The proof of this lemma is given in [2, Lemma 2.1] for the 3-D case with uniform triangulation and in [6] for general ones. Its simplification for the 2-D case can be found, e.g., in [7, Lemma 3.1] and [10, the proof of Lemma 1].

Remark 6.3. We note that we will apply a slight generalisation of the lemma above which is also covered by the literature. Specifically, inequality (6.11) remains true if $v_{\ell, i_{m}}$ is an arbitrary $L_{2}$-function on $\gamma_{m}$.

\subsection{2. $\ell_{2}$ Inequalities}

In the following, we denote by $\ell_{2}(\mathcal{N})$ the space of tuples over an index set $\mathcal{N}$. Here, $\mathcal{N}$ takes the values $\overline{\mathcal{N}}_{\ell, j}, \overline{\mathcal{N}}_{\ell}$, and $\mathcal{N}_{\ell, j}, \mathcal{N}_{\ell}$. The appropriate scaling of the $\ell_{2}$-norms regarding the equivalence (2.6) is

$$
\left\|\underline{v}_{\ell}\right\|_{\ell_{2}(\mathcal{N})}:=\sqrt{\sum_{p \in \mathcal{N}} h_{\ell}^{2}\left|\underline{v}_{\ell}(p)\right|^{2}} \quad \text { for } \mathcal{N}=\mathcal{N}_{\ell, j}, \overline{\mathcal{N}}_{\ell, j}, \mathcal{N}_{\ell} \text {, and } \overline{\mathcal{N}}_{\ell} .
$$

Here, $\underline{v}_{\ell}(p)=v_{\ell}\left(x_{p}\right)$ is the notation for the $p$-component of the vector $\underline{v}_{\ell}$ according to Remark 6.1.

The well-known equivalence between $\ell_{2}$ and $L_{2}$ norms is first stated for the space $X_{\ell}$. Note that we have assumed quasi-uniformity of the triangulations in each $\Omega_{j}$, $j \in I$.

Lemma 6.4. (a) Let $\bar{\phi}_{\ell, j}$ be the isomorphism from $\ell_{2}\left(\overline{\mathcal{N}}_{\ell, j}\right)$ onto $S_{\ell, j}\left(\Omega_{j}\right)$ (cf. (2.5)). Then there is a constant $C$ independent of $j$ and the mesh size $h_{\ell}$ such that

$$
\frac{1}{C}\left\|\underline{v}_{\ell, j}\right\|_{\ell_{2}\left(\overline{\mathcal{N}}_{\ell, j}\right)} \leq\left\|\bar{\phi}_{\ell, j}\left(\underline{v}_{l, j}\right)\right\|_{L_{2}\left(\Omega_{j}\right)} \leq C\left\|\underline{v}_{\ell, j}\right\|_{\ell_{2}\left(\overline{\mathcal{N}}_{\ell, j}\right)} \quad \text { for } \underline{v}_{\ell, j} \in \ell_{2}\left(\overline{\mathcal{N}}_{\ell, j}\right) \text {. }
$$

(b) Similarly, for $\bar{\phi}_{\ell}$ being the isomorphism between $\ell_{2}\left(\overline{\mathcal{N}}_{\ell}\right)$ and $X_{\ell}(\Omega)$, we have

$$
\frac{1}{C}\left\|\underline{v}_{\ell}\right\|_{\ell_{2}\left(\overline{\mathcal{N}}_{\ell}\right)} \leq\left\|\bar{\phi}_{\ell}\left(\underline{v}_{\ell}\right)\right\|_{L_{2}(\Omega)} \leq C\left\|\underline{v}_{\ell}\right\|_{\ell_{2}\left(\overline{\mathcal{N}}_{\ell}\right)} \quad \text { for } \underline{v}_{\ell} \in \ell_{2}\left(\overline{\mathcal{N}}_{\ell}\right) .
$$

Since $\mathcal{N}_{\ell, j} \subseteq \overline{\mathcal{N}}_{\ell, j}$, the following estimate is trivial:

$$
\left\|\underline{v}_{\ell}\right\|_{\ell_{2}\left(\mathcal{N}_{\ell}\right)} \leq\left\|\underline{v}_{\ell}\right\|_{\ell_{2}\left(\overline{\mathcal{N}}_{\ell}\right)} .
$$

The opposite inequality is obviously not valid for coefficient vectors $\underline{v}_{\ell} \in \ell_{2}\left(\overline{\mathcal{N}}_{\ell}\right)$ belonging $v_{\ell} \in X_{\ell}(\Omega)$. However, it is true if $v_{\ell} \in V_{\ell}(\Omega)$, as stated in

Lemma 6.5. Let $\phi_{\ell}: \underline{v}_{\ell} \in \ell_{2}\left(\mathcal{N}_{\ell}\right) \mapsto v_{\ell}=\sum_{p \in \mathcal{N}_{\ell}} \underline{v}_{\ell}(p) b_{\ell, p} \in V_{\ell}(\Omega)$ with basis functions from Section 6.1 .2 be the isomorphism from $\ell_{2}\left(\mathcal{N}_{\ell}\right)$ onto $V_{\ell}(\Omega)$. 
Let $\underline{x}_{\ell}:=\bar{\phi}_{\ell}^{-1} v_{\ell} \in \ell_{2}\left(\overline{\mathcal{N}}_{\ell}\right)$ be the coefficient vector for $v_{\ell} \in X_{\ell}(\Omega) \supset V_{\ell}(\Omega)$. Then we have $\underline{x}_{\ell}(p)=\underline{v}_{\ell}(p)$ for all vertices $p \in \mathcal{N}_{\ell}$ and

$$
\left\|\underline{x}_{\ell}\right\|_{\ell_{2}\left(\overline{\mathcal{N}}_{\ell}\right)} \leq C\left\|\underline{v}_{\ell}\right\|_{\ell_{2}\left(\mathcal{N}_{\ell}\right)} .
$$

Proof: Given a slave edge $\delta:=\delta_{m} \subset \bar{\Omega}_{j_{m}}$ and the corresponding master edge $\gamma:=\gamma_{m} \subset \bar{\Omega}_{i_{m}}$, let $A:=A_{m}$ and $B:=B_{m}$ be the endpoints of $\delta$. The proof of (6.14) reduces to show that for each $\delta=\delta_{m} \subset \Gamma, m \in \mathcal{M}$,

$$
\left\|\underline{x}_{\ell}\right\|_{\ell_{2}(\overline{\mathcal{N}} \ell \cap \delta)} \leq C\left\|\underline{v}_{\ell}\right\|_{\ell_{2}\left(\mathcal{N}_{\ell} \cap(\bar{\gamma} \cup A \cup B)\right)} .
$$

Here $\overline{\mathcal{N}}_{\ell} \cap \delta$ is the set of interior nodal points of $\delta$ which by the definition of $\Pi_{\ell, m}$ depend on $\left.\mathcal{N}_{\ell} \cap(\bar{\gamma} \cup A \cup B)\right)$. Now define $w_{\ell} \in L_{2}\left(\Gamma_{i_{m}, j_{m}}\right)$ by $w_{\ell} \in S_{\ell, m, j_{m}}$ and

$$
w_{\ell}= \begin{cases}v_{\ell} & \text { at the nodal points } A \text { and } B, \\ 0 & \text { at the nodal points of } \delta .\end{cases}
$$

Roughly speaking $w_{\ell}$ is obtained from $\left.v_{\ell}\right|_{\delta}$ by the restriction to the values on $\partial \delta$. Note that $v_{\ell, i_{m}}-w_{\ell}$ and $v_{\ell, j_{m}}-w_{\ell}$ also satisfy the mortar condition (6.4). With this we have $\left.\left(v_{\ell, j_{m}}-w_{\ell}\right)\right|_{\delta}=\Pi_{m, \ell}\left(v_{\ell, i_{m}}-w_{\ell}, 0\right)$. It follows from Lemma 6.2 that

$$
\left\|v_{\ell}\right\|_{L_{2}(\delta)} \leq\left\|w_{\ell}\right\|_{L_{2}(\delta)}+C\left(\left\|v_{\ell}\right\|_{L_{2}(\gamma)}+\left\|w_{\ell}\right\|_{L_{2}(\gamma)}\right) .
$$

After rewriting this in terms of the coefficients, the proof of (6.15) is complete.

Proposition 6.6. The isomorphism $\phi_{\ell}$ from $\ell_{2}\left(\mathcal{N}_{\ell}\right)$ onto $V_{\ell}(\Omega)$ satisfies

$$
\frac{1}{C}\left\|\underline{v}_{\ell}\right\|_{\ell_{2}\left(\mathcal{N}_{\ell}\right)} \leq\left\|\phi_{\ell}\left(\underline{v}_{\ell}\right)\right\|_{L_{2}(\Omega)} \leq C\left\|\underline{v}_{\ell}\right\|_{\ell_{2}\left(\mathcal{N}_{\ell}\right)} \quad \text { for all } \underline{v}_{\ell} \in \ell_{2}\left(\mathcal{N}_{\ell}\right) .
$$

Proof: Set $v_{\ell}:=\phi_{\ell}\left(\underline{v}_{\ell}\right)$ and extend the vector $\underline{v}_{\ell}$ by the components of $\underline{x}_{\ell}:=$ $\bar{\phi}_{\ell}^{-1} v_{\ell}$ at $p \in \overline{\mathcal{N}}_{\ell} \backslash \mathcal{N}_{\ell}$. Combination of (6.13) and (6.12) yields

$$
\left\|\underline{v}_{\ell}\right\|_{\ell_{2}\left(\mathcal{N}_{\ell}\right)} \leq C\left\|v_{\ell}\right\|_{L_{2}(\Omega)} \text {. }
$$

From (6.12d (6.14) we obtain the final part

$$
\left\|v_{\ell}\right\|_{L_{2}(\Omega)} \leq C\left\|\underline{v}_{\ell}\right\|_{\ell_{2}\left(\overline{\mathcal{N}}_{\ell}\right)} \leq C^{2}\left\|\underline{v}_{\ell}\right\|_{\ell_{2}\left(\mathcal{N}_{\ell}\right)} .
$$




\subsection{Choice of $\Sigma, S, \sigma$, and $\pi$}

In the diagram below, $V_{\ell-1}$ and $V_{\ell}$ are the mortar spaces introduced in Section 6.1.1. The coefficient spaces $\mathcal{U}_{j}=\ell_{2}\left(\mathcal{N}_{j}\right),(j=\ell-1, \ell)$, together with their isomorphisms $\phi_{j}$ have already been specified.

$$
\begin{aligned}
& \Sigma=X_{\ell} \stackrel{\sigma}{\longrightarrow} S=\mathcal{U}_{\ell}=\ell_{2}\left(\mathcal{N}_{\ell}\right) \\
& \text { inclusion } \uparrow \nwarrow \downarrow \pi=\phi_{\ell} \\
& V_{\ell-1} \underset{\iota}{\longrightarrow} V_{\ell} \\
& \phi_{\ell-1} \uparrow \quad \uparrow \phi_{\ell} \\
& \mathcal{U}_{\ell-1} \underset{p}{\longrightarrow} \mathcal{U}_{\ell}
\end{aligned}
$$

We choose $\Sigma:=X_{\ell}$ equipped with the norm of $L_{2}(\Omega)$. By construction $V_{\ell}=$ $V_{\ell}(\Omega) \subset X_{\ell}$ holds. Since $X_{\ell-1} \subset X_{\ell}$, also $V_{\ell-1} \subset X_{\ell}$. Hence, the sum $V_{\ell}+$ $V_{\ell-1}$ is contained in $X_{\ell}$.

The space $S$ is chosen as $S:=\mathcal{U}_{\ell}=\ell_{2}\left(\mathcal{N}_{\ell}\right)$. The obvious choice for $\pi$ is $\pi:=\phi_{\ell}$. Injectivity and boundedness of $\pi$ is given by Proposition 6.6.

The essential part is the choice of the mapping $\sigma: X_{\ell} \rightarrow \ell_{2}\left(\mathcal{N}_{\ell}\right)$. Given a patchwise continuous function $v$ from $X_{\ell}$ and indices $p=\left(x_{p}, \Omega_{i(p)}\right) \in \mathcal{N}_{\ell}$, we set

$$
(\sigma v)(p):=v\left(x_{p}\right)
$$

according to Remark 6.1. Then $\sigma v=((\sigma v)(p))_{p \in \mathcal{N}_{\ell}}$ is the coefficient vector in $\ell_{2}\left(\mathcal{N}_{\ell}\right)$.

Remark 6.7. $\sigma: X_{\ell} \rightarrow \ell_{2}\left(\mathcal{N}_{\ell}\right)$ is bounded uniformly in $\ell$ :

$$
\left\|\sigma v_{\ell}\right\|_{\ell_{2}\left(\mathcal{N}_{\ell}\right)} \leq C\left\|v_{\ell}\right\|_{L_{2}(\Omega)} .
$$

Proof: Set $\underline{w}_{\ell}:=\sigma v_{\ell}$. The combination of (6.13) and (6.12) yields

$$
\left\|\underline{w}_{\ell}\right\|_{\ell_{2}\left(\mathcal{N}_{\ell}\right)}=\left\|\underline{v}_{\ell}\right\|_{\ell_{2}\left(\mathcal{N}_{\ell}\right)} \leq C\left\|v_{\ell}\right\|_{L_{2}(\Omega)} .
$$

We note that the opposite inequality $\left\|v_{\ell}\right\|_{L_{2}(\Omega)} \leq C\left\|\underline{v}_{\ell}\right\|_{\ell_{2}\left(\mathcal{N}_{\ell}\right)}$ is not valid for all $v_{\ell} \in V_{\ell}+V_{\ell-1} \subset \Sigma=X_{\ell}$. There are nontrivial functions $u_{\ell}$ in $V_{\ell}+V_{\ell-1}$ with $\sigma\left(u_{\ell}\right)=0$. For its construction take a function $v_{\ell-1}$ with support in $\Omega_{i}$ and set $v_{\ell}:=v_{\ell-1}$. Extend $v_{\ell-1}$ and $v_{\ell}$ to the slave side according to the different mortar 
conditions of level $\ell$ and $\ell-1$, respectively. Then the function $u_{\ell}:=v_{\ell}-v_{\ell-1}$ vanishes on $\Omega_{i}$, but it is nonzero on the slave side. After a slight modification at nodal points neighboured to the slave nodes, one obtains zero coefficients for all $p \in \mathcal{N}_{\ell}$, but $u_{\ell} \neq 0$. Thus, $\sigma$ is not injective.

Remark 6.8. $\iota=\phi_{\ell} \circ \sigma$ is a projection onto $V_{\ell}$.

Proof: Given $v_{\ell} \in V_{\ell}, \underline{v}_{\ell}:=\sigma v_{\ell}$ are the values at the nodal points of $\mathcal{N}_{\ell}$. Because of the Lagrange basis property, $\phi_{\ell} \underline{v}_{\ell}$ recovers the function $v_{\ell} \in V_{\ell}$.

So far, we have ensured the conditions (3.2)-(3.4) required in Section 3.2.

Finally, we observe that inequality (5.1) follows from

$$
\left|a_{\ell}\left(u_{\ell}, v_{\ell}\right)\right| \leq C\left\|u_{\ell}\right\|_{H_{\text {broken }}^{1}}\left\|v_{\ell}\right\|_{H_{\text {broken }}^{1}}
$$

with $\left\|v_{\ell}\right\|_{H_{\text {broken }}^{1}}^{2}:=\sum_{j \in I}\left\|\left.v_{\ell}\right|_{\Omega_{j}}\right\|_{H^{1}\left(\Omega_{j}\right)}^{2}$ and the standard inverse inequality

$$
\left\|\left.v_{\ell}\right|_{\Omega_{j}}\right\|_{H^{1}\left(\Omega_{j}\right)} \leq C h_{\ell}^{-1}\left\|\left.v_{\ell}\right|_{\Omega_{j}}\right\|_{L_{2}\left(\Omega_{j}\right)} \quad \text { for } v_{\ell} \in S_{\ell, j}\left(\Omega_{j}\right) .
$$

Hence, if the underlying second order boundary value problem satisfies the solvability and regularity conditions, all necessary requirements for the two- and multigrid convergence are satisfied.

\section{Geometrically Nonconforming Case}

In this section, we discuss a discretisation of the problem (6.1) on non-matching triangulations in the case when the subdomains $\Omega_{j}$ of the decomposition $\bar{\Omega}=$ $\bigcup_{j \in I} \bar{\Omega}_{j}$ do not form a 'triangulation' of $\Omega$. In our case the $\Omega_{j}$ 's are assumed to be polygons, and this means that a vertex of $\Omega_{j}$ is not necessarily a vertex of its neighbours $\Omega_{i}$. This case is called the geometrically nonconforming case in the mortar method, and a typical situation is depicted in Fig 1.

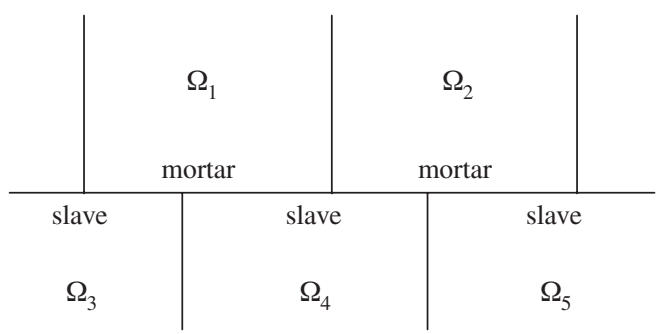

Figure 1. Detail of a geometrically nonconforming mesh 
We first formulate a discrete problem for (6.1) and then extend our treatment of the geometrically conforming case to this one.

\subsection{Discrete Problem}

To formulate the discrete problem, we will adapt some of our previous notations and introduce some new ones. Let here $\Gamma_{j, k}, k=1, \ldots, K_{j}$, denote the edges of $\Omega_{j}$ and $K_{j}$ be its number. Let $\Gamma=\bigcup_{j \in I} \partial \Omega_{j} \backslash \partial \Omega$ be as in (6.2). We select a family of edges, say $\left\{\gamma_{k}, k \in K\right\}$, from the set of all edges $\left\{\Gamma_{j, k}: j \in I, 0 \leq k \leq K_{j}\right\}$ satisfying the condition

$$
\bigcup_{k \in K} \bar{\gamma}_{k}=\bar{\Gamma}, \quad \gamma_{k} \cap \gamma_{k^{\prime}}=\emptyset \text { if } k \neq k^{\prime} .
$$

These edges are called masters (mortars). The edges $\Gamma_{j, k}$ which are not masters are called slaves (nonmortars) and denoted by $\delta_{m}, m \in \mathcal{M}$. Note that the nonmortars $\left\{\delta_{m}\right\}$ satisfy a condition similar to (7.1):

$$
\bigcup_{m \in \mathcal{M}} \bar{\delta}_{m}=\bar{\Gamma}, \quad \delta_{m} \cap \delta_{m^{\prime}}=\emptyset \text { if } m \neq m^{\prime}
$$

We adapt the notation $\delta_{m}\left(\gamma_{k}\right)$ as an edge of $\Omega_{j_{m}}\left(\Omega_{i_{k}}\right)$. A mortar space $M_{\ell, m}\left(\delta_{m}\right)$ is defined on each $\delta_{m}$ in the same way as in Section 6.

We say that a function $v_{\ell} \in X_{\ell}(\Omega)$ satisfies the mortar condition on an edge $\delta_{m}$ if

$$
\int_{\delta_{m}}\left[v_{\ell}\right] \psi d s=0 \quad \text { for all } \psi \in M_{\ell, m}\left(\delta_{m}\right)
$$

where [ $v_{\ell}$ ] is the jump of $v_{\ell}$. The discretisation space $V_{\ell}(\Omega)$ for (6.1) is defined as the subspace of $X_{\ell}(\Omega)$ of functions satisfying the mortar conditions (7.2) for all $m \in \mathcal{M}$.

We are now able to formulate the discrete problem. Find $u_{\ell} \in V_{\ell}(\Omega)$ such that

$$
a_{\ell}\left(u_{\ell}, v_{\ell}\right)=f\left(v_{\ell}\right) \quad \text { for all } v_{\ell} \in V_{\ell} .
$$

The problem has a unique solution, and the error is bounded by

$$
\left\|u_{\ell}-u\right\|_{L_{2}(\Omega)} \leq C h_{\ell}^{2} \sum_{j \in I}\|u\|_{H^{2}(\Omega)},
$$

where $u_{\ell}$ and $u$ are the solutions of (7.3) and (6.1), respectively. The proof of the error estimates proceeds as in the geometrically conforming case. Specifically the extension of the stability results is based on Remark 6.3. The estimates of the consistency errors are local in nature and carry over without changes. 
Our goal is to design and analyse the multigrid method for solving (7.3) using the previous scheme.

\subsection{Matrix Form}

We rewrite (7.3) in the matrix form using that

$$
V_{\ell}=\operatorname{span}\left\{b_{\ell, p}: p \in \mathcal{N}_{\ell}\right\}
$$

The basis functions $b_{\ell, p}$ are defined again by the Lagrange basis property similar to those in Section 6. For $p \in \mathcal{N}_{\ell}$ with $x_{p}$ lying in the interior of $\Omega_{j_{p}}, b_{\ell, p}$ is the same as in Section 6. For $x_{p} \in \gamma_{m}$, where $\gamma_{m}$ is a master edge in $\bar{\Omega}_{j(p)}, b_{\ell, p}$ is defined by

$$
b_{\ell, p}= \begin{cases}\varphi_{\ell, p} & \text { in } \bar{\Omega}_{j(p)} \\ \prod_{\ell, n}\left(\varphi_{\ell, p}, 0\right) & \text { on } \delta_{n}\end{cases}
$$

where $\delta_{n}$, the slave edge of $\Omega_{j_{n}}$, is such that $\emptyset \neq \delta_{n} \cap \gamma_{m} \subset \operatorname{supp}\left(\varphi_{\ell, p}\right)$; on the remaining vertices in $\bar{\Omega}_{j_{n}}$ and the remaining substructures $b_{\ell, p}(x)$ is extended by zero. For $x_{p}$ which are vertices of $\Omega_{i_{p}}, b_{\ell, p}$ are defined in the same way as in Section 6. Here, the extension by the mortar projection is understood as in (7.2).

Using $b_{\ell, p}$, we obtain

$$
A_{\ell} \underline{u}_{\ell}=\underline{f}_{\ell} .
$$

The matrix $A_{\ell}$ is symmetric and positive definite and its eigenvalues satisfy also the inequalities (6.10).

\subsection{Two-Grid and Multigrid Method}

We comment here on the two-grid method for (7.3) (or (7.4)). This is sufficient to analyse the multigrid method.

Since $\ell$ is a variable level number, the space $V_{\ell-1}$ and the mortar projection $\Pi_{\ell-1, m}$ are defined as well. We first formulate the counterpart of Lemma 6.2 for the geometrically nonconforming case. Let $\delta_{m}$ be a slave edge of $\Omega_{j_{m}}$ and let $v_{\ell, j_{m}} \in$ $S_{\ell, j_{m}}\left(\Omega_{j_{m}}\right)$ be the components of $v_{\ell}=\left\{v_{\ell, j}\right\} \in V_{\ell}(\Omega)$. By the definition

$$
v_{\ell, j_{m} \mid \delta_{m}}=\Pi_{\ell, m}\left(\tilde{v}_{\ell}, \operatorname{Tr}_{m} v_{\ell, j_{m}}\right)
$$

where $\tilde{v}_{\ell \delta_{m}}=\sum_{k} v_{\ell, i_{k} \mid \delta_{m} \cap \gamma_{k}}$ and the sum is taken over $v_{i_{k}}$ defined on $\bar{\Omega}_{i_{k}}$ involving $\gamma_{k}$ with $\delta_{m} \cap \gamma_{k} \neq \emptyset$. 
Lemma 7.1. For $v_{\ell}=\left\{v_{\ell, j}\right\}_{j \in I} \in V_{\ell}$ on $\delta_{m} \subset \bar{\Omega}_{j_{m}}$ with $\operatorname{Tr}_{m} v_{j_{m}}=0$ holds

$$
\left\|v_{\ell, j_{m}}\right\|_{L_{2}\left(\delta_{m}\right)}^{2} \leq C \sum_{k}\left\|v_{\ell, i_{k}}\right\|_{L_{2}\left(\gamma_{k} \cap \delta_{m}\right)}^{2}
$$

where $v_{\ell, j_{m}}$ is defined by (7.5) and $C$ is a constant independent of $h_{\ell, i_{k}}$.

For a proof of this lemma, see [2, Lemma 2.1].

Using this lemma, we check that Lemmata 6.2, 6.4 and 6.5 from Section 6 are also valid for the discussed case. Defining $\sigma, \pi$ and $\iota=\sigma \circ \pi$ as in the previous section, we check that $\pi$ is injective and bounded, $\sigma$ is bounded and $\iota$ is a projection. Thus the two-grid method for (7.3) (and (7.4)) is uniformly convergent with respect to $h_{\ell}$.

\section{References}

[1] Ben Belgacem, F.: The mortar element method with Lagrange multipliers. Numer. Math. (to appear).

[2] Ben Belgacem, F., Maday, Y.: The mortar element method for three dimensional finite elements. RAIRO M² AN 31, 289-302 (1997).

[3] Bernardi, C., Maday, Y., Patera, A. T.: A new nonconforming approach to domain decomposition: the mortar element method. In: Nonlinear partial differential equations and their applications (Brezis, H., Lions, J. L., eds.), pp. 13-51. New York: Pitman 1994.

[4] Braess, D.: Finite elements: Theory, fast solvers, and applications in solid mechanics. Cambridge: Cambridge University Press 1997.

[5] Braess, D., Verfürth, R.: Multi-grid methods for nonconforming finite element methods. SIAM J. Numer. Anal. 27, 979-986 (1990).

[6] Braess, D., Dahmen, W.: Stability estimates of the mortar finite element method for 3-dimensional problems. East-West J. Numer. Math. 6, 249-264 (1998).

[7] Braess, D., Dahmen, W., Wieners, C.: A multigrid algorithm for the mortar finite element method. SIAM J. Numer. Anal. (to appear).

[8] Brenner, S.: An optimal order multigrid method for $P 1$ nonconforming finite elements. Math. Comput. 32, 1-15 (1989).

[9] Dryja, M.: An iterative substructuring method for elliptic mortar finite element problems with discontinuous coefficients. In: Domain decomposition methods 10 (Mandel, J., Farhat, Ch., Cai, X-C., eds.), pp. 94-103. Contemporary Mathematics, vol. 218.

[10] Dryja, M.: Additive Schwarz methods for elliptic mortar finite element problems. In: Modeling and optimization of distributed parameter systems, applications to engineering (Malanowski, K., Nahorski, Z., Peszynska, M., eds.), pp. 31-50. London: Chapman \& Hall 1996.

[11] Gopalakrishnan, J., Pasciak, J. E.: Multigrid for the mortar finite element method. Report ISC-9804-MATH, Texas A\&M University, 1998.

[12] Hackbusch, W.: Multi-grid methods and applications. Berlin Heidelberg New York: Springer 1985.

[13] Hackbusch, W.: Elliptic differential equations. Theory and numerical treatment. Berlin Heidelberg New York Tokyo: Springer 1992. 
[14] Stevenson, R.: The Cascade iteration for nonconforming discretisations. Report 9803 University of Nijmegen, Feb. 1998.

[15] Brenner, S.: Convergence of nonconforming multigrid methods without full elliptic regularity. Mat. Comp. 68, 25-53 (1999).

D. Braess

Fakultät für Mathematik

Ruhr-Universität

D-44780 Bochum

Germany

e-mail: braess@num.ruhr-uni-bochum.de
M. Dryja

Department of Mathematics

Warsaw University

Banacha 2

02-097 Warsaw

Poland

e-mail: dryja@hydra.mimuw.edu.pl

W. Hackbusch

Max-Planck-Institut

Mathematik in den Naturwissenschaften

Inselstr. 22-26

D-04103 Leipzig

Germany

e-mail: wh@mis.mpg.de 